\title{
Stage I Hepatocellular Carcinoma AJCC v8
}

National Cancer Institute

\section{Source}

National Cancer Institute. Stage I Hepatocellular Carcinoma AJCC v8. NCI Thesaurus.

Code C134516.

Stage I includes: IA (T1a, N0, M0); IB (T1b, N0, M0). T1a: Solitary tumor equal to or less

than $2 \mathrm{~cm}$. T 1 b: Solitary tumor larger than $2 \mathrm{~cm}$ without vascular invasion. N0: No

regional lymph node metastasis. M0: No distant metastasis. (AJCC 8th ed.) 